Int. J. Dev. Biol. 56: 351-356 (2012)

doi: $10.1387 / \mathrm{ijdb} .123514 \mathrm{sm}$

\title{
KDEL tagging: a method for generating dominant-negative inhibitors of the secretion of TGF- $\beta$ superfamily proteins
}

\author{
SHINYA MATSUKAWA ${ }^{1}$, YUKI MORIYAMA ${ }^{2}$, TADAYOSHI HAYATA ${ }^{3}$, HARUKA SASAKI ${ }^{1}$, YUZURU ITO ${ }^{4}$, \\ MAKOTO ASASHIMA4 and HIROKI KURODA ${ }^{1,2, *}$

\begin{abstract}
${ }^{1}$ Department of Education (Sciences), Shizuoka University, ${ }^{2}$ Graduate School of Science and Technology, Shizuoka University, Shizuoka, ${ }^{3}$ Department of Molecular Pharmacology, Medical Research Institute, Tokyo Medical and Dental University, Tokyo and ${ }^{4}$ Research Center for Stem Cell Engineering, National Institute of Advanced Industrial Science and Technology (AIST), Tokyo, Japan
\end{abstract}

\begin{abstract}
Most endoplasmic reticulum (ER)-retained proteins contain a carboxy-terminal signal sequence called the ER retention signal motif such as the Lys-Asp-Glu-Leu (KDEL) motif. Using this molecular mechanism, we developed a new dominant-negative assay, designated the KDEL-tag trap assay, to negatively regulate secretion of disulfide bond-dependent protein dimers, as typified by TGF- $\beta$ superfamily proteins. First, we tested this method on the Nodal protein Xnr5, which is a well-studied mesoderm inducer in vertebrates. Tagging of Xnr5 protein with KDEL at the carboxyterminus effectively blocked the secretion of Xnr5, resulting in complete inhibition of mesoderm induction in Xenopus embryogenesis. Second, we examined the usefulness of the KDEL-tag trap assay on BMPs, which are well-known negative regulators of neural induction and ventralizing factors during early development, and demonstrated that the functions of the BMP family proteins BMP4 and ADMP were blocked by the KDEL-tag trap assay. Moreover, the technical feasibility of the KDEL-tag trap assay was confirmed in a cell culture system using mouse osteoblasts. Taken together, these results suggest that the KDEL-tag trap assay can be adapted to inhibit a variety of plasma membrane or secreted proteins of a multimeric nature.
\end{abstract}

KEY WORDS: KDEL, ER retention signal, Nodal, BMP, TGF- $\beta$ superfamily

\section{Introduction}

In eukaryotic cells proteins destined for the secretory pathway have a 5-15-amino acid-long hydrophobic fragment, also known as a signal sequence, at the amino-terminal end (Blobel, 1980). This signal sequence allows the proteins to be translocated into the ER lumen, and the proteins exported from the ER then enter the first cis-cisterna of the Golgi apparatus. In the Golgi apparatus, further posttranslational modification occurs, and the trans-cisterna of the Golgi apparatus, which contains ready-to-use proteins, forms the trans-Golgi network and secretory granules that eventually fuse with the plasma membrane (Fig. 1A). However, some proteins do not have the ability to traffic to the extracellular space, although they contain a signal sequence at the amino-terminal end. Many ER-retained proteins such as chaperones and folding enzymes contain a carboxy-terminal signal sequence designated the ER retention signal motif Lys-Asp-Glu-Leu (KDEL) (Munro et al., 1987). In eukaryotes, for example, calreticulin (Crt), protein disulfide isomerase (PDI), and the 78-kDa and 94-kDa of glucose-regulated proteins (GRP78 and GRP94) are retained in the ER by the KDEL sequence. Yeasts and plants use His-Asp-Glu-Leu (HDEL) instead of KDEL, and a few other related sequences, including KEEL in ERp72, HNEL in the LDL-receptor associated protein, HSEL in Canopy1 (Hirate et al., 2006), and HDEF in Calumenin have been described (reviewed in Capitani et al., 2009). Interestingly, all of these KDEL-like sequences seem to act in an almost identical manner, and thus, they might be interchangeable. More importantly, they enable ER proteins to bind to a seven-transmembrane-spanning protein that is considered as a KDEL receptor, and known to cycle between the ER and Golgi apparatus (Fig. 1A, B).

The transforming growth factor- $\beta$ (TGF- $\beta$ ) superfamily consists of a large number of structurally related, dimeric proteins that are

Abbreviations used in this paper: BMP, bone morphogenetic protein; Crt, calreticulin; ER, endoplasmic reticulum; KDEL, Lys-Asp-Glu-Leu motif; TGF, transforming growth factor.

\footnotetext{
*Address correspondence to: Hiroki Kuroda. Department of Education (Sciences), Shizuoka University, 836 Ohya, Suruga-ku, Shizuoka 422-8529, Japan. Tel: +81-54-238-4304. Fax: +81-54-287-6693. e-mail: ehkurod@ipc.shizuoka.ac.jp - web: http://www.ipc.shizuoka.ac.jp/ ehkurod
} 
secreted into the extracellular matrix. They act as either hormones, growth factors, or paracrine mediators, to regulate various types of biological events in animals, including cell proliferation and differentiation, developmental patterning and morphogenesis, and disease pathogenesis (Massagué, 1998). Because of their biological and medical importance, developing a method capable of effectively inhibiting the activities of TGF- $\beta$ superfamily molecules has always been a goal of many cell biologists. Currently, several assays are in use: a small molecule called dorsomorphin selectively blocks bone morphogenetic protein (BMP) type I receptors (Yu et al., 2008); the skeletal integrity of mice is improved by adding the type II activin receptor soluble extracellular domain (Pearsall et al., 2008), and the secretion of Xnr5 is blocked by a cleavage mutant of Xnr5 (Onuma et al., 2005).

In this study, we introduce a novel and simple approach for inhibiting TGF- $\beta$ superfamily proteins, which are first synthesized

A

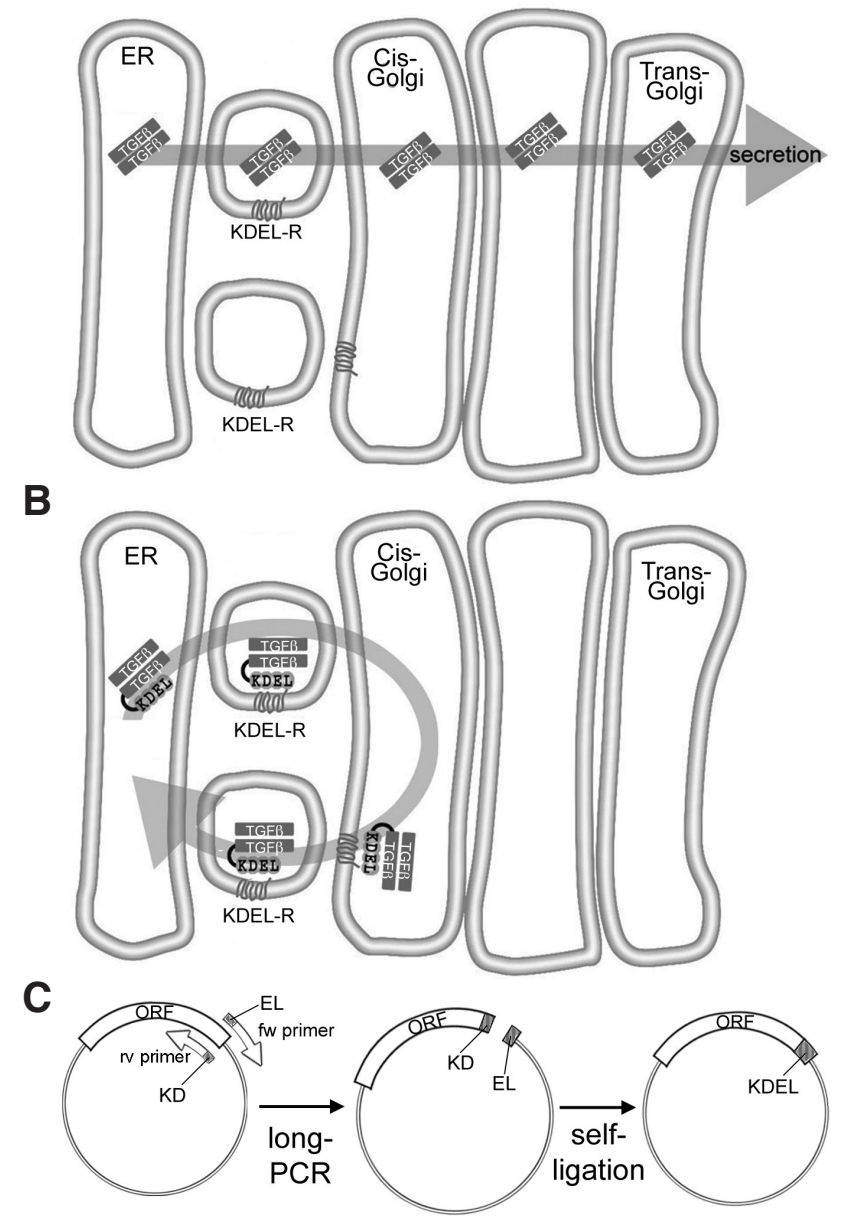

Fig. 1. Schematic representation of the KDEL-tag trap assay. (A) NoFmal pathway of secreted homodimer proteins such as TGF- $\beta$ superfamily proteins. (B) Scheme of the KDEL-tag trap assay. An experimentally generated carboxy-terminal KDEL-conjugated subunit may bind subunits in the complementary endogenous heterodimeric protein via the formation of a disulfide bond. The resultant protein complex should then be trapped by the KDEL receptor (KDEL-R), resulting in its continuous shuttling between the ER and Golgi apparatus. (C) A method to conjugate KDEL to the carboxy-terminal end of target molecules. Only a single long-range polymerase chain reaction is required. as larger precursor proteins containing a propeptide region that is removed prior to secretion, and a mature region that is dimerized by disulfide bonds to attain biological activity when the protein is secreted (Kingsley, 1994). On the basis of this phenomenon, we hypothesized that the artificially KDEL-conjugated TGF- $\beta$ superfamily proteins may dimerize with their endogenous counterparts. If this occurred, the target TGF- $\beta$ superfamily molecules would bind to the KDEL receptor, which would confine them inside the cell, with the resultant protein complex shuttling between the ER and Golgi apparatus. These trapped TGF- $\beta$ superfamily proteins would not be secreted, and would, therefore, be absent from the extracellular space (Fig. 1B). Thus, here, we show that this, indeed, is the case, and that KDEL-tagged subunits are potent dominantnegative reagents. Therefore, the KDEL-tag trap assay could be widely applicable to inhibit the function of multimeric secreted or plasma membrane proteins.

\section{Experimental Procedures}

To conjugate the KDEL motif to the carboxy-terminal end of a target protein, six nucleotides encoding EL were added to the 5 end of the forward primer, followed by a stop codon sequence, while KD was added to the reverse primer (Fig. 1C). Plasmids pCS2-Xnr5 and both pCS2-BMP4 and pCS2-ADMP, used for creating KDEL-tag mutants, have been described in previous reports (Takahashi et al., 2000; Reversade et al., 2005). The primers 5' GAACTCTAACTCGAGCCTCTAGAACT 3' and 5' GTCCTTATAACATCCACATTCCTCTAC3' were used for constructing Xnr5-KDEL. The primers 5' GAACTCTGAGTCTGAGATCCAAACAAAA 3' and 5' GTCCTTACGGCACCCACACCCA 3' were used for constructing BMP4-KDEL. The primers 5' GAACTCTAACTCGAGCCTCTAGAACT 3' and 5' GTCCTTGTGGCACCCGCAG 3' were used for constructing ADMP-KDEL. The underlined sequences in the primers were used for adding KD or EL. We performed long-length polymerase chain reaction involving these primers and high-fidelity DNA polymerase (KOD-plus Neo, Toyobo, Japan). The 5' ends of the DNA fragments were phosphorylated using T4 polynucleotide kinase, followed by self-ligation with T4 DNA ligase (Fig. 1C). The mRNAs used for microinjection experiments were transcribed from linearized plasmids by using an mMessage mMachine kit (Ambion).

\section{Results \& Discussion}

\section{The KDEL-tag trap assay blocks Nodal signaling}

Only one polymerase chain reaction is required to conjugate the four KDELamino acids at the carboxy-terminal end of a target protein (Fig. 1C). To evaluate whether the mutated molecules constructed by this simple method actually function, we used our experimental model Xenopus Nodal-related 5 (Xnr5), a TGF- $\beta$ superfamily Nodal protein that functions during Xenopusembryogenesis. Nodal proteins, which belong to the TGF- $\beta$ superfamily, are known to be essential for mesoderm inductions during vertebrates embryonic development (De Robertis et al., 2004; De Robertis, 2009). In the Xenopus species, Xnr5 is expressed on the dorsal-vegetal side of blastula embryos prior to any other players in mesoderm inductions (Takahashi et al., 2000). Therefore, the mesoderm would fail to develop if the secretion step in Xnr5 synthesis were to be inhibited using the KDEL-tag trap assay. To study the location of Xnr5 protein during the early developmental stages in Xenopus 
A

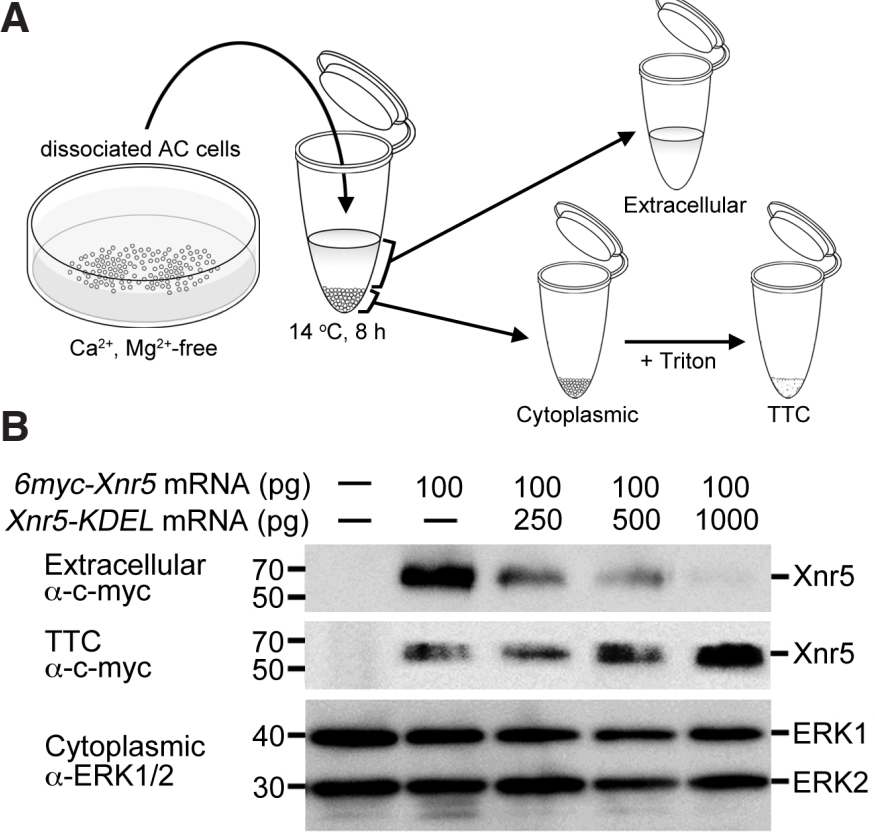

Fig. 2. KDEL-conjugated Nodal protein inhibits the secretion of epitopetagged Nodal protein. (A) Diagram illustrating the preparation of protein solutions. Animal caps were cut from embryos injected with mRNAs, dissociated in calcium- and magnesium-free solution (CMF), cultured for 8 $h$ at $14{ }^{\circ} \mathrm{C}$ in $\mathrm{CMF}$, and centrifuged at 1,000 $\times \mathrm{g}$ for $1 \mathrm{~min}$. Extracellular or cytoplasmic fractions were prepared from the supernatant or precipitate. The Triton X-100-treated cytoplasmic (TTC) fraction, comprising proteins inside the $E R$, was generated by adding Triton-X100 to a final concentration of $1 \%$ (middle panel). (B) Translocation of myc-tagged Xnr5 protein from the extracellular space (top fraction) to the ER space (middle fraction) following the addition of Xnr5-KDEL. ERK1/2 in the intracellular fraction was examined as a positive control (bottom).

the yolk plug region was expanded, and the blastopore appeared slightly irregular (Fig. 3B). In case of high amounts of Xnr5-KDEL, the ventro-lateral blastopore was not formed, and only a short, pigmented line was observed on the dorsal side (Fig. 3C). In addition, the expression of Xbrachyury (Xbra) (Smith et al., 1991), which is a pan-mesodermal marker expressed at the gastrula stage, was completely suppressed by the KDEL-tag trap assay for Xnr5 (Fig. 3D, E). In later stages, mesodermal and axial defects were induced by Xnr5-KDEL mRNA in a dose-dependent manner (Fig. $3 \mathrm{~F}-\mathrm{H}$ ). Those phenotypes induced by the KDEL-tag trap assay were very similar to the effects caused by Cerberus-short (Cer-S) embryos, we injected myc-tagged Xnr5mRNA with or withoutKDEL-conjugated Xnr5(Xnr5-KDEL) mRNA into these embryos, cut presumptive ectodermal regions generally called animal caps $(A C)$, and collected secreted proteins from the supernatant, cytoplasmic proteins from the harvested cell pellet, or proteins solubilized using Triton X-100 detergent in the cellular fraction (Fig. 2A). As expected, the secretion of Xnr5 protein was inhibited by Xnr5-KDEL in a dose-dependent manner (Fig. 2B, top). It was also confirmed that the nonsecreted Xnr5 protein remained in the cell pellet (Fig. $2 \mathrm{~B}$, middle), while the positive control cytoplasmic ERK1/2 was not affected (Fig. 2B, bottom). In addition, visualization of the protein fractions secreted on the SDSPAGE gel by using Coomassie blue showed that Xnr5-KDEL did not change the amounts of other kinds of secreted proteins (data not shown). These biochemical results indicated that the KDEL-tag trap assay worked during the secretion process in a target-specific manner.

We next investigated whether Xnr5-mediated mesoderm induction might be inhibited by this assay. In amphibian embryos the pigmented blastopore on the surface of the dorsal-vegetal side appears as the first visible sign of mesoderm formation in the early gastrula stage. The blastopore then extends to the ventral side, and becomes a circle by the mid-gastrula stage (Fig. 3A). The size of this circle, whose inside is generally known as the yolk plug, is gradually reduced during gastrulation movements by the process called epiboly, and is eventually closed by the beginning of the neurula stage. As expected, this blastopore formation was influenced by the KDEL-tag trap assay for Xnr5. In the embryos injected with a low amount of Xnr5-KDEL mRNA,
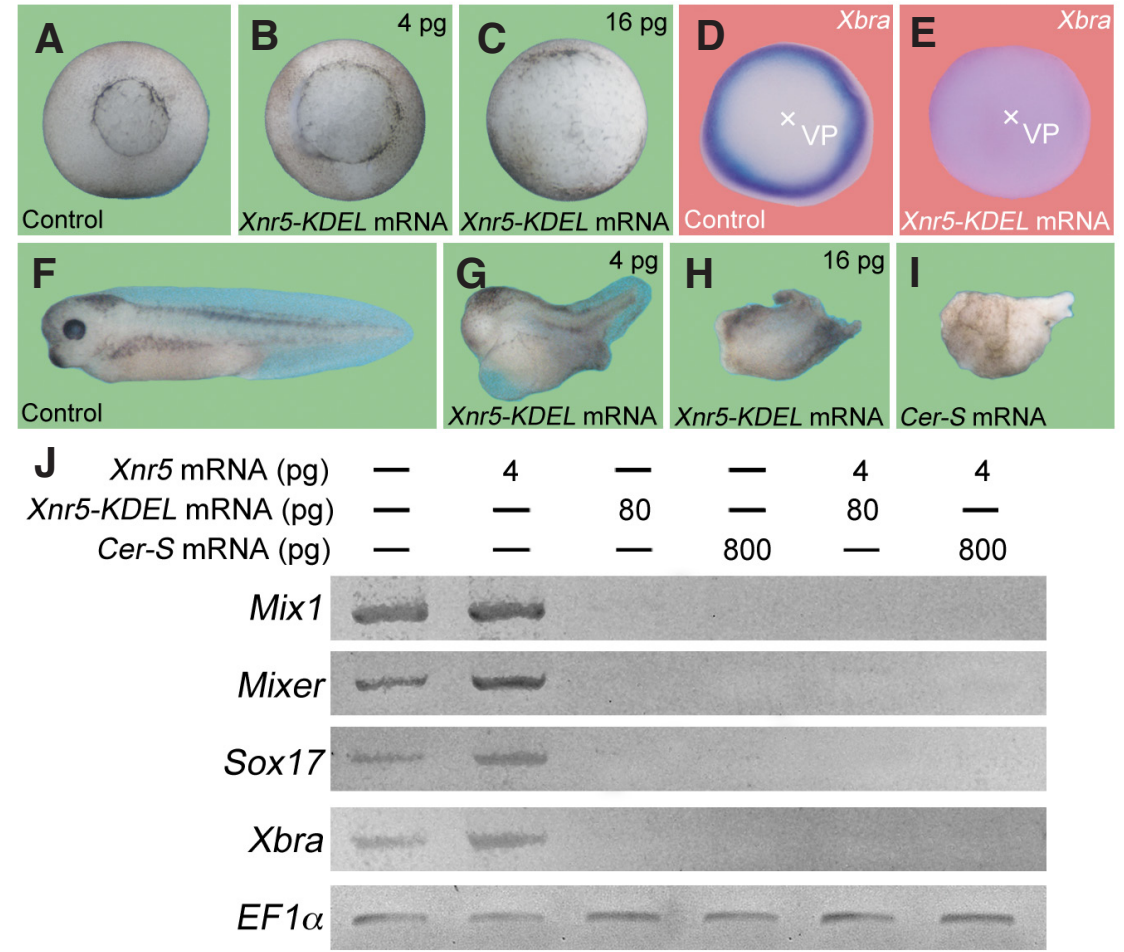

Fig. 3. Mesoderm formation is inhibited by microinjection of Xnr5-KDEL mRNA. (A-E) Inhibition of mesoderm formation by using microinjection of Xnr5-KDEL mRNA (4 or 16 pg), as demonstrated by monitoring the presence of defects in the blastopore, as well as by using in situ hybridization analysis for the pan-mesodermal marker Xbra in the embryos at stage 11 of embryogenesis. (F-I) Resultant Cer-S mRNA-mediated phenotypic effects on the embryos that fail to develop mesoderm (shown in I) were also confirmed in embryos at later stages, as shown in (G,H). (J) The expression of all Nodal target markers such as Mix1, Mixer, Sox17, and Xbra was blocked following microinjection of Xnr5-KDEL mRNA. EF1 $\alpha$ was used as a positive control. Total mRNA was prepared from the embryos at stage 9 of embryogenesis. 
(Piccolo et al., 1999), a truncated form of Cerberus that functions as a specific Nodal antagonist (Fig. 3l, compare to 3H). Specific inhibition of Xnr5 can also be provided by a cleavage mutant of Xnr5, which lacks the RXXR cleavage site for the convertase proteases located between the propeptide and mature domains (Onuma et al., 2005)); despite this, the efficiency of the inhibitory action of the KDEL-tag trap assay is almost 30 times higher than
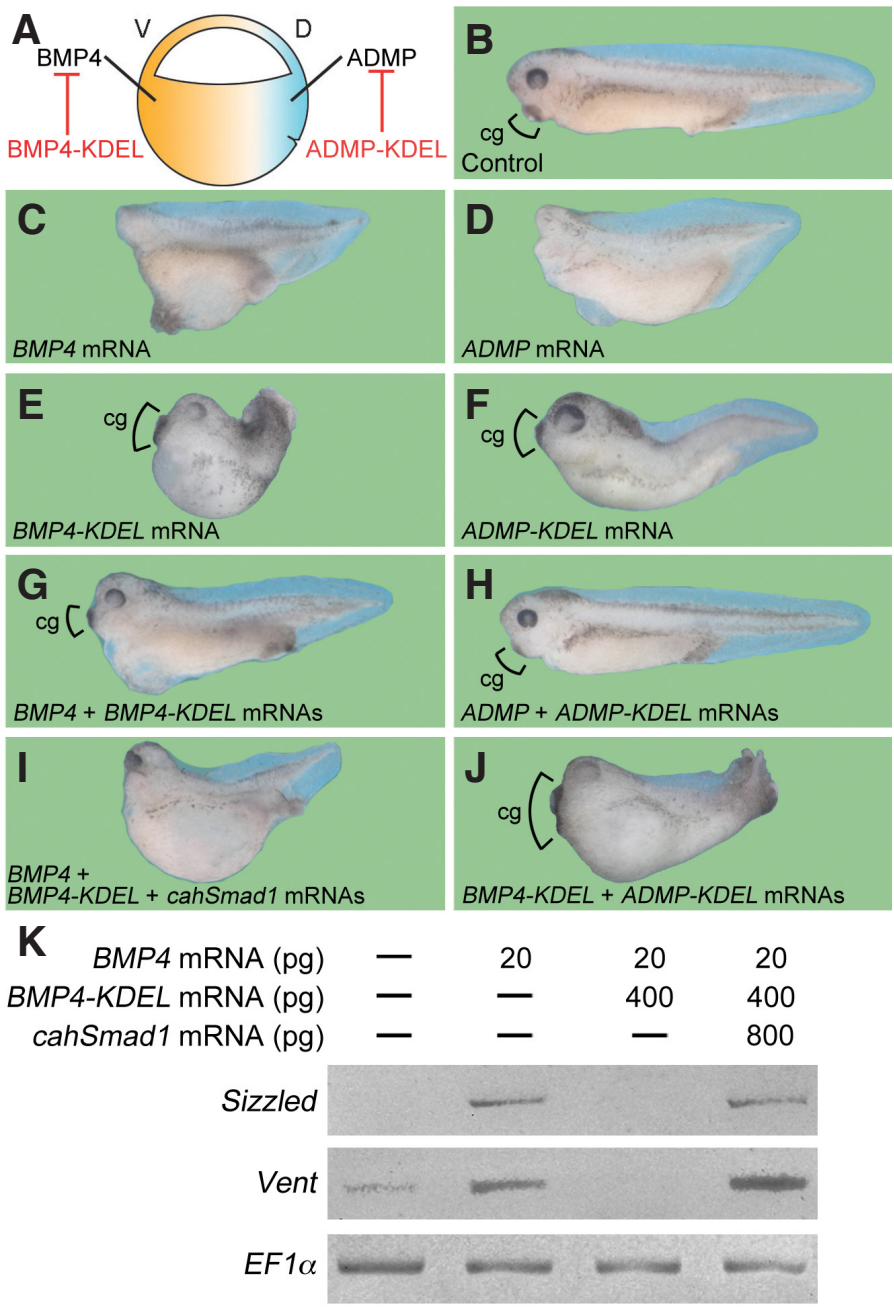

Fig. 4. Effect of the KDEL-tag trap assay on BMP signaling. (A) Schematic diagram showing ventrally expressed BMP protein known as BMP4, and dorsally expressed ADMP in the early gastrula embryonic stage. (B-F) Microinjections of the mRNA encoding KDEL-conjugated BMP4 (400 pg) or ADMP $(400 \mathrm{pg})$ caused typical phenotypic effects of low-BMPactivity, such as expansion of the cement gland (cg), while posteriorization was induced by microinjections of the MRNA encoding wild-type BMP4 or ADMP (each 20 pg). (G,H) The effects of KDEL-conjugated BMPs were rescued by the overexpression of wild-type mRNAs. (I) Microinjection of mRNA encoding a constitutively active form of human SMAD1 (cahSMAD1, 400 pg) overcame the rescue effect as shown in $G$, indicating that the effects of KDEL-conjugated molecules occur upstream of SMAD1. (J) The anti-BMP effects increased with the co-injection of both mRNAs than with separate injections of the individual KDEL-traps. (K) Expression of the ventral BMP target markers Sizzled and Vent was blocked by the microinjection of BMP4-KDEL MRNA, and rescued by the co-injection of mRNA encoding cahSMAD1. EF1 $\alpha$ was used as a loading control. that of Cer-S, and 60 times higher than a cleavage type of mutant. To experimentally obtain complete inhibition of mesoderm formation as shown in Fig. $3 \mathrm{H}$, which was induced by $16 \mathrm{pg}$ of Xnr5-KDEL mRNA, more than 500 pg of Cer-S mRNA or 1 ng of mRNA encoding a cleavage mutant of $\mathrm{Xnr} 5$ are usually required (Piccolo et al., 1999; Onuma et al., 2005). This suggests that the proteins conjugated with KDEL at the the carboxy-terminally might bind wild-type molecules with very high affinity and interfere with the normal pathway of secretion of protein dimers to the extracellular space. Moreover, similar to the effects of Cer-S, we found that the expression of major Nodal target genes such as Mix1, Mixer, Sox17, and Xbra was inhibited by the co-injection of Xnr5-KDEL mRNA (Fig. 3J). These results demonstrate that the KDEL-tag trap assay is very effective at inhibiting Nodal activity during Xenopus embryogenesis. In these experiments it was unclear whether other Nodal proteins such as Xnr1, Xnr2, Xnr4, and Xnr6 were also targeted by $X n r 5-K D E L$; nevertheless, its strong inhibitory effects suggest that this is highly possible.

An HDEL sequence is the other major version of ER retention signals, and it is broadly used in plants and yeasts (Capitani et al., 2009). Phenotypical analysis in Xenopus demonstrated that almost the same events reported above for Xnr5-tagged KDEL occurred with Xnr5 that had been tagged with the HDEL motif (data not shown). In zebrafish, both Canopy1-HSEL, which is the endogenous signal in Canopy1-mediated regulation of FGF signaling, and the experimentally generated Canopy1-KDEL (Hirate and Okamoto, 2006) have similar functions, as was the case in our study. This suggests that KDEL variants might function identically in the vertebrate secretory pathway.

\section{The KDEL-tag trap assay is applicable to BMPs}

BMPs were originally discovered on the basis of their ability to induce bone and cartilage formation, and are now known to mediate various biological events (Reddi, 1997). In total, 12 of BMP-like molecules are members of the TGF- $\beta$ superfamily and have been well studied as central players in neural formation and dorsoventral patterning during early embryogenesis (De Robertis et al., 2004; De Robertis, 2009). We, therefore, tried to identify whether the KDEL-tag trap assay was adaptable to BMPs. In vertebrates, spatially opposed expression of the BMP family proteins BMP4 and antidorsalizing morphogenetic protein (ADMP), which is most closely related to human BMP3, can promote restoration of dorsovent of ventral structures during Xenopus embryogenesis. As shown in Fig. 4A, we designed BMP4-KDEL and ADMP-KDEL to block ventral and dorsal BMP signaling, respectively. As previously reported (Moos et al., 1995; Reversade et al., 2005), the microinjection of normal BMP4 or ADMP mRNA in our studies induced ventralization in Xenopus embryos (Fig. 4 B-D). In contrast, both $B M P 4-K D E L$ and $A D M P-K D E L$ mRNA did not induce ventralization (Fig. 4 E,F). Instead, BMP4-KDEL mRNA caused strong dorsalization with big cement gland formation, which is a feature of dorsalization (Fig. 4E). ADMP-KDEL mRNA also induced weaker dorsalization, albeit in a weaker manner BMP4-KDEL, with minor expansion of the cement gland (Fig. 4F). Similar to Nodal proteins, cleavage mutants for BMP have also been reported (Hawley et al., 1995), but our method seems to be more effective. It is likely that entire ligand family proteins such as BMP2 and BMP7 were inhibited using our method. We also confirmed that the effects of the KDEL-conjugated proteins were rescued by co-injecting the 


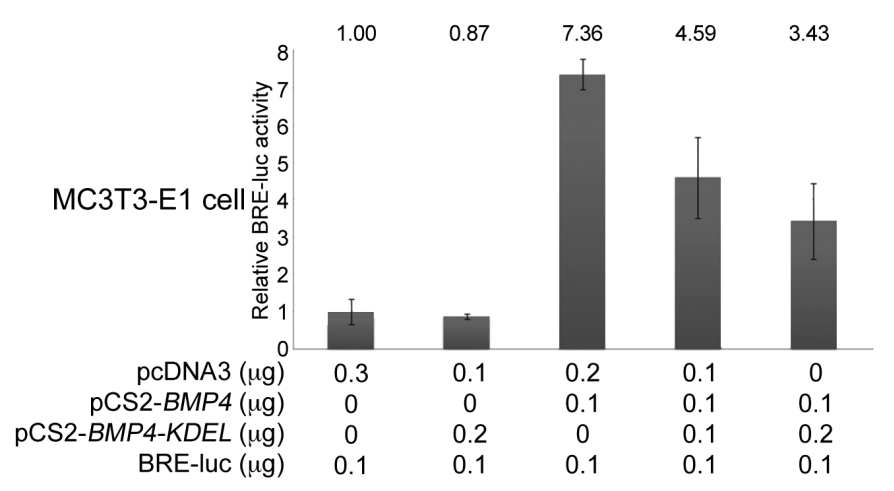

Fig. 5. BMP4-KDEL inhibits BMP4-induced transcriptional activation in a concentration-dependent manner on mouse MC3T3-E1 osteoblast cells. In all cases, plasmids were transfected with $0.1 \mu \mathrm{g}$ of BRE-luc (pOLuc-xld3-BRE) for the luciferase assay. pcDNA3 was used for adjusting the total amounts of transfected DNA to $0.4 \mu \mathrm{g}$. Luciferase activity increased following the transfection of PCS2-BMP4, but it was reduced by co-transfection with pCS2-BMP4-KDEL in a dose-dependent manner.

tagged protein with mRNA encoding wild-type protein (Fig. $4 \mathrm{G}, \mathrm{H}$ ). Moreover, we showed that co-injection with mRNA encoding a constitutively-active form of human SMAD1 (cahSMAD1) overcame all upstream effects of BMP4 and BMP4-KDEL, resulting in normal ventralization with small head (Fig. 4I). The cahSMAD1 protein has phosphorylation-resistant mutations in its SMAD1 linker region, and is not affected by ERK/MAPK and GSK3, or phospho-mimetic mutations of SMAD1, in which C-terminal SVS phosphorylation sites are mutated into EVE (Fuentealba et al., 2007). Moreover, the expression of BMP target genes such as Sizzled and Ventwas blocked by BMP4-KDEL and rescued by cahSMAD1 mRNA (Fig. $4 \mathrm{~K})$. As expected, co-injection of BMP4-KDEL and ADMP-KDEL mRNAs into all four blastomeres at the four-cell stage induced the formation of bigger cement gland and stronger dorsalization (Fig. 4J) compared to the induced phenotypes, following separate injections of either BMP4-KDEL or ADMP-KDEL (Fig. 4 E,F).

Finally, we wished to determine whether this system also worked in other cell systems, because the roles of BMP signaling in cultured cells have been extensively studied in recent years by using loss-of-function assays (Yu et al., 2008; Pearsall et al., 2008). When mouse MC3T3-E1 osteoblast cells were transfected with a plasmid encoding the BMP-responsive element fused to the firefly luciferase reporter (BRE-luc) gene (von Bubnoff et al., 2005), and stimulated by cotransfection with pCS2-BMP4, or a combination of pCS2-BMP4 and pCS2-BMP4-KDEL, we observed significant inhibition of the BMP signal with BMP4-KDEL (Fig. 5). These results indicate that the KDEL-tag trap assay might be applicable to various cell biological systems.

In the present study, we have demonstrated a novel, yet simple and very effective technique for generating dominant-negative proteins, by conjugating the KDEL motif to the carboxy-terminal end of TGF- $\beta$ superfamily proteins amino acid sequences. In this system, we used only one long-range polymerase chain reaction requiring two primers, each having six additional nucleomers, for adding the $\mathrm{K}-\mathrm{D}$ or $\mathrm{E}-\mathrm{L}$ motifs (Fig. $\mathrm{C}$ ). Since many studies have been focused on Nodal and BMP proteins in general, the reagents described in this study will be, at a minimum, useful to cell biologists who study these molecules. Furthermore, as we confirmed our technique by using both microinjection and cell culture assays, it may also be applicable in studies involving different molecules in various organisms. In future, it will be particularly interesting to determine whether our assay can block the trafficking of multimeric plasma membrane receptors to the cell surface. The uses of this KDEL-tag trap assay should, therefore, allow researchers in molecular and cellular biology to readily generate new tools for inhibiting the activities of target dimeric or polymeric secreted proteins or membrane receptors.

\section{Acknowledgements}

We thank Professor Shuji Takahashi, Yoshiki Sasai, Jim Smith, and Camille Bouissou for providing DNA constructs; Miduki Nishimura, Tsubasa Mishiku, and Nana Saito for providing technical assistance, and Shoko Mori and Yoshihisa Ohata for their valuable comments on the manuscript. This research was supported by a grant-in-aid received from the Shizuoka Research Institute.

\section{References}

BLOBEL, G. (1980). Intracellular protein topogenesis. Proc. Natl. Acad. Sci. USA 77: 1496-1500.

CAPITANI, M., SALLESE, M. (2009). The KDEL receptor: new functions for an old protein. FEBS Lett 583: 3863-3871.

DEROBERTIS, E.M. (2009). Spemann's organizer and the self-regulation of embryonic fields. Mech Dev 126: 925-941.

DE ROBERTIS, E.M., KURODA, H. (2004). Dorsal-ventral patterning and neura induction in Xenopus embryos. Annu Rev Cell Dev Biol 20: 285-308.

FUENTEALBA, L.C., EIVERS, E., IKEDA, A., HURTADO, C., KURODA, H., PERA, E., DE ROBERTIS, E.M. (2007). Integrating patterning signals: Wnt/GSK3 regulates the duration of the BMP/Smad1 signal. Cell 131: 980-993.

HAWLEY, S.H., WÜNNENBERG-STAPLETON, K., HASHIMOTO, C., LAURENT, M.N., WATABE, T., BLUMBERG, B.W., CHO, K.W. (1995). Disruption of BMP signals in embryonic Xenopus ectoderm leads to direct neural induction. Genes Dev 9: 2923-2935.

HIRATE, Y., OKAMOTO, H. (2006). Canopy1, a novel regulator of FGF signaling around the midbrain-hindbrain boundary in zebrafish. Curr Biol 16: 421-427.

KINGSLEY, D.M. (1994). The TGF-beta superfamily: new members, new receptors, and new genetic tests of function in different organisms. Genes Dev 8: 133-146.

MASSAGUÉ, J. (1998). TGF-beta signal transduction. Annu Rev Biochem67: 753-791.

MOOS, JR.M., WANG, S., KRINKS, M. (1995). Anti-dorsalizing morphogenetic protein is a novel TGF-beta homolog expressed in the Spemann organizer. Development 121: 4293-4301.

MUNRO, S., PELHAM, H.R. (1987). A C-terminal signal prevents secretion of luminal ER proteins. Cell 48: 899-907.

ONUMA, Y., TAKAHASHI, S., HARAMOTO, Y., TANEGASHIMA, K., YOKOTA, C., WHITMAN, M., ASASHIMA, M. (2005). Xnr2 and Xnr5 unprocessed proteins inhibit Wnt signaling upstream of dishevelled. Dev Dyn 234: 900-910.

PEARSALL, R.S., CANALIS, E., CORNWALL-BRADY, M., UNDERWOOD, K.W., HAIGIS, B., UCRAN, J., KUMAR, R., POBRE, E., GRINBERG, A., WERNER, E.D., GLATT, V., STADMEYER, L., SMITH, D., SEEHRA, J., BOUXSEIN, M.L. (2008). A soluble activin type IIA receptor induces bone formation and improves skeletal integrity. Proc Natl Acad Sci USA 105: 7082-7088.

PICCOLO, S., AGIUS, E., LEYNS, L., BHATTACHARYYA, S., GRUNZ, H., BOUWMEESTER, T., DE ROBERTIS, E.M. (1999). The head inducer Cerberus is a multifunctional antagonist of Nodal, BMP and Wnt signals. Nature 397: 707-710.

REDDI, A.H. (1997). Bone morphogenetic proteins: an unconventional approach to isolation of first mammalian morphogens. Cytokine Growth Factor Rev. 8: 11-20.

REVERSADE, B., DE ROBERTIS, E.M. (2005). Regulation of ADMP and BMP2/4/7 at opposite embryonic poles generates a self-regulating morphogenetic field. Cell 123: 1147-1160.

SMITH, J.C., PRICE, B.M., GREEN, J.B., WEIGEL, D., HERRMANN, B.G. (1991) Expression of a Xenopus homolog of Brachyury $(\mathrm{T})$ is an immediate-early response to mesoderm induction. Cell 67: 79-87.

TAKAHASHI, S., YOKOTA, C., TAKANO, K., TANEGASHIMA, K., ONUMA, Y., GOTO 
J., ASASHIMA, M. (2000). Two novel nodal-related genes initiate early inductive events in Xenopus Nieuwkoop center. Development 127: 5319-5329.

VON BUBNOFF, A., PEIFFER, D.A., BLITZ, I.L., HAYATA, T., OGATA, S., ZENG, Q., TRUNNELL, M., CHO, K.W. (2005). Phylogenetic footprinting and genome scanning identify vertebrate BMP response elements and new target genes. Dev
Biol 281: 210-216.

YU, P.B., DENG, D.Y., HONG, C.S., CUNY, G.D., BOUXSEIN, M.L., HONG, D.W. MCMANUS, P.M., KATAGIRI, T., SACHIDANANDAN, C., KAMIYA, N., FUKUDA, T., MISHINA, Y., PETERSON, R.T., BLOCH, K.D. (2008). BMP type I receptor inhibition reduces heterotopic [corrected] ossification. Nat Med 14: 1363-1369.

\section{Further Related Reading, published previously in the Int. J. Dev. Biol.}

Inhibitory Smads and bone morphogenetic protein (BMP) modulate anterior photoreceptor cell number during planarian eye regeneration

Alejandro González-Sastre, $\mathrm{M}^{\mathrm{a}}$ Dolores Molina and Emili Saló

Int. J. Dev. Biol. (2012) 56: 155-163

Bmp4-directed nuclear cyan fluorescent protein provides a tool for live imaging and reveals cellular resolution of Bmp4 expression patterns during embryogenesis Chuan-Wei Jang, Liang Gao, Mary E. Dickinson and Richard R. Behringer Int. J. Dev. Biol. (2010) 54: 931-938

Expression of Bmp ligands and receptors in the developing Xenopus retina Jennifer C. Hocking and Sarah McFarlane

Int. J. Dev. Biol. (2007) 51: 161-165

A change in response to Bmp signalling precedes ectodermal fate choice

Chris T. Dee, Abigail Gibson, Andrea Rengifo, Shun-Kuo Sun, Roger K. Patient and Paul J. Scotting

Int. J. Dev. Biol. (2007) 51: 79-84

Xantivin suppresses the activity of EGF-CFC genes to regulate nodal signaling Kousuke Tanegashima, Yoshikazu Haramoto, Chika Yokota,Shuji Takahashi and Makoto Asashima

Int. J. Dev. Biol. (2004) 48: 275-283

5 yr ISI Impact Factor $(2010)=2.961$

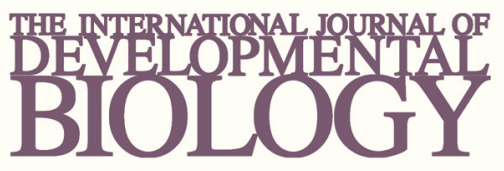

Volume 54 Nos. $6 / 7$
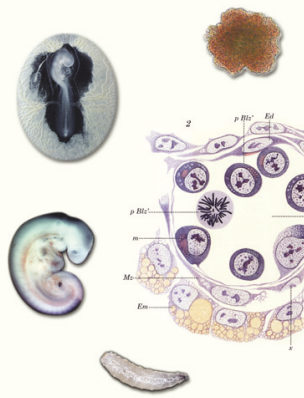

(2) (8) (8)
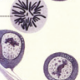

(1)
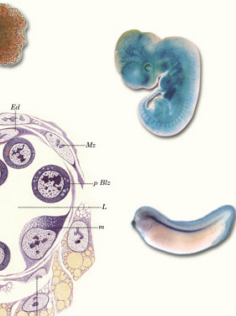

Developmental Hematopoiesis

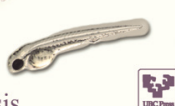

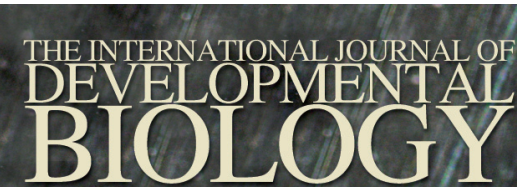
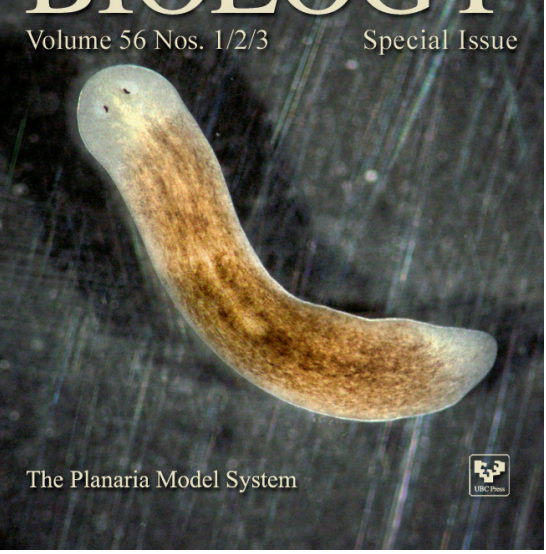
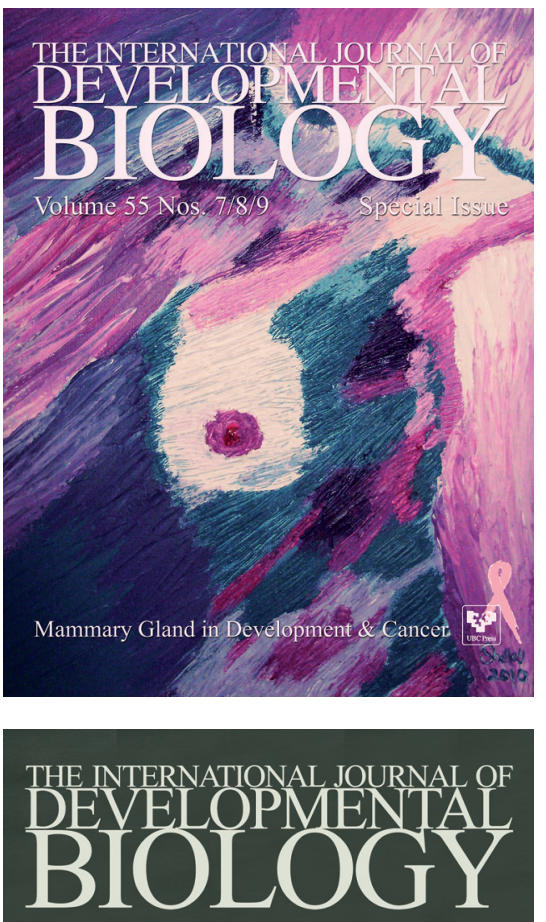

Volume 55 Nos. $4 / 5$

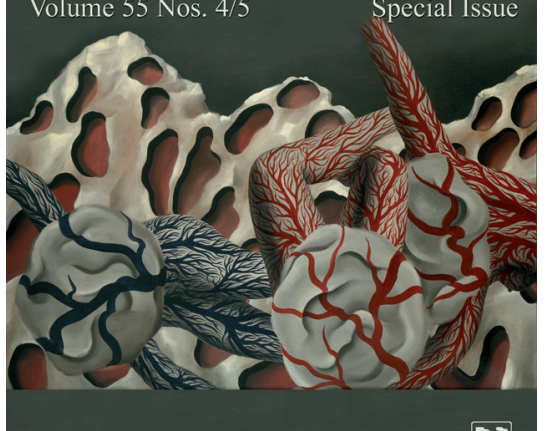

Angiogenesis in Development \& Cancer 